\title{
Evaluation of MU2net as an Online Secondary Dose Check for MR Guided Radiation Therapy with the Elekta Unity MR Linac
}

John Baines

Townsville Hospital

Ariadne Shoobridge ( $\square$ ariadne.shoobridge@health.qld.gov.au )

Townsville Hospital

\section{Research Article}

Keywords: secondary dose calculation, Elekta Unity, MR-Linac, MU2net

Posted Date: May 18th, 2021

DOl: https://doi.org/10.21203/rs.3.rs-368526/v1

License: (c) (1) This work is licensed under a Creative Commons Attribution 4.0 International License.

Read Full License 


\section{Abstract}

During the adaptive workflow associated with MRgRT, a secondary dose calculation is required and MU2net (DOSIsoft, France) is one commercial option. The suitability of MU2net to be used in conjunction with the online Monaco treatment planning system of the Elekta Unity (Elekta AB, Stockholm, Sweden), is evaluated in this work. Monaco and MU2net point doses are compared for various fields on and off axis and at different SSDs. To investigate the comparative effects of attenuation due to the cryostat, couch and posterior coil, measured, MU2net and Monaco dose outputs at the isocentre, as a function of gantry angle, were compared. Point doses for the beams of nine step and shoot IMRT (SSIMRT) test plans (courtesy Elekta) were calculated with Monaco v5.4 and compared to corresponding doses computed with MU2net. In addition, Monaco v5.4 and MU2net point doses were compared for 1552 beams treated on the Unity at our facility. For the on-axis fields investigated the agreement between MU2net and measured data is acceptable. MU2net and Monaco point doses for the Elekta SSIMRT test plans were within $\pm 5.0 \%$ and $\pm 6.4 \%$ for beams delivered from gantry zero and at planned beam angles, respectively. For the 1552 beams delivered approximately $80.0 \%$ of MU2net and Monaco point doses agree within $\pm 5.0 \%$, therefore it is recommended to correlate MU2net Dose Reference Points (DRPs )with pre and post treatment dosimetry verification. Computational accuracy of MU2net could be enhanced with improved modelling of attenuation due to the couch, cryostat and posterior MR imaging coil.

\section{Introduction}

In December 2019 the Townsville University Hospital Cancer Centre introduced an Elekta Unity MR-linac (Elekta AB, Stockholm, Sweden) to clinical use, the first such cancer treatment system in the southern hemisphere. This linac combines radiation therapy with high soft-tissue contrast MR imaging and facilitates adaptive radiotherapy using real time Graphical Processing Unit (GPU) based Monte Carlo treatment planning (Monaco v5.4) [1-4]. The Monte Carlo dose calculation algorithm (GPUMCD) incorporates radiation transport in the presence of a magnetic field and makes use of GPUs, facilitating parallel processing which reduces calculation times [5].

The MR linac basic structure in Fig. 1 consists of a beam generation system that is mounted on a vertically orientated rotating annulus. The annulus rotates at $6.0 \mathrm{rpm}$ delivering a $7 \mathrm{MV} \mathrm{FFF}$ beam directed to the centre of rotation within the bore of the Unity. A modified Ingenia 1.5 T superconducting magnet is mounted such that each of its two superconducting coils sit on either side of the rotating annulus. The magnet cryostat containing liquid helium is located within the annulus (see Fig. 1) and radiation beams must traverse the cryostat before entering the bore. Consequently the angular dependence of the cryostat attenuation needs to be characterised and modelled in the treatment planning system [6]. With this configuration $\mathrm{B}_{0}$ is perpendicular to the axis of rotation of the $\mathrm{x}$-ray source for all beam directions [7]. The source to isocentre distance is $143.5 \mathrm{~cm}$, the bore diameter is $70.0 \mathrm{~cm}$ and the couch is $14.0 \mathrm{~cm}$ below the isocentre. There is no collimator rotation and the maximum field size in the isocentric plane is $57.4 \mathrm{~cm}$ crossplane, (IEC61217 x-direction) and $22.0 \mathrm{~cm}$ inplane, (IEC61217 ydirection). The multileaf collimator is based on the Elekta Agility model with 80.0 leaf pairs with rounded 
leaf ends, each with a projected width of approximately $7.18 \mathrm{~mm}$ at isocentre. Leaf motion occurs parallel to the $y$-axis which is also the only direction of motion for the couch. Since the couch direction of movement is perpendicular to the plane of rotation of the $x$-ray source, only coplanar treatments are possible and the current treatment options are conformal fields and step and shoot IMRT.

The adaptive treatment planning options available with Monaco v5.4 are broadly divided into two main workflows, namely Adapt to Position (ATP) and Adapt to Shape (ATS). For further details of both ATP and ATS the reader can refer to Winkel et al [8]. With adaptive planning, it is critical to have an independent online dosimetric evaluation of each adapted plan, a secondary MU/dose check, before proceeding to treat. For the clinical cases considered in this work ATP was exclusively used.

At our facility we evaluated the use of MU2net software (DOSIsoft, France) for monitor unit (MU) verification with the Elekta Unity MR linac. The commissioning of this dose calculation engine is based on MPPG5a [9] recommended tests and is the subject of an internal report. In this work we report on output factors (OFs) for on axis square fields and off-axis customised fields, the variation of output with gantry angle and treatment plan point dose comparisons between MU2net and Monaco v5.4.

The dose computation in MU2net is divided into two successive steps: reconstruction of the beam fluence at the exit of the treatment head and dose computation in the medium [10]. The beam fluence map is reconstructed according to the imported DICOM RT Plan data. For each irradiation segment, i.e. between two successive control points (CPs), the calculation considers the open FFF beam profile modified by the leaf arrangement (which acts as a layer). It takes into account the respective leaf/jaw transmission factor and the leaf penumbra shape by applying a gaussian filter. The fluence map is expressed in terms of MU given from the RT Plan file for each irradiation segment. This MU data is corrected for head-scatter effects ( $S c$ component or $\mathrm{OF}_{\text {air }}$ of the output factor) to report the variations of the dose rate with the beam aperture. The total fluence map is reconstructed from the whole set of CPS and this representation of the beam intensity modulation is applied onto a large parallelepiped water phantom for dose calculation. The dose engine separates a primary dose calculation considering a simple exponential attenuation function of the radiological depth, and a scatter dose reconstructed with a Clarkson's sector summation algorithm. When imported into MU2net, DICOM RT StructureSet files are used to extract radiological depths from a reconstructed anatomical patient model aligned on the treatment couch. MU2net is a dose point comparison solution, designed for fast computation time and ease of implementation. In practice MU2net point dose comparisons with Monaco v5.4 are achieved by assigning DRPs to each beam of a Monaco plan and exporting the Monaco plan (StructureSet and Total Plan Dose) to MU2net. MU2net is accessed via a web-based interface and resides on a virtual server (16.0 MB CPU). Once accessed, MU2net launches the dose computation at the DRPs for each beam and compares them automatically to corresponding TPS point doses. A single DRP can be selected for all beams but generally individual DRPs for each beam is preferable. It should be noted that Monaco exports point dose DRP values to MU2net. However, for comparison of corresponding DRPs the mean dose within a sphere of radius $3.0 \mathrm{~mm}$ for each Monaco DRP has been used in this work. MU2net is only a 
verification system for point doses, it cannot be used like a conventional TPS. An alternative comparable secondary MU check, RadCalc (Lifeline Software Inc., Tyler, USA), is described by Graves et al [11].

Our department has successfully introduced MU2net as a secondary dose check. To the best of our knowledge this is the first report dedicated to the use of this software in the adaptive planning workflow of an Elekta Unity system.

\section{Materials And Methods}

\section{Model configuration}

The MU2net dose engine is modelled using depth dose, output factors and profiles for the 7 MV FFF photon beam of the Unity, in a B = 0 T environment (courtesy Elekta, Crawley, UK). The output of the Elekta Unity in our department is set to $1.000 \mathrm{~Gy} / 100 \mathrm{MU}(\mathrm{B}=1.5 \mathrm{~T})$ at the isocentre, depth of $5.0 \mathrm{~cm}$ in water, SSD $=138.5 \mathrm{~cm}$, gantry $90.0^{\circ}$, for a $10.0 \times 10.0 \mathrm{~cm}^{2}$ field. These are also the conditions associated with the calibration point for Monaco v5.4. At a depth of $10.0 \mathrm{~cm}$, gantry $0.0^{\circ}$, the specified dose calibration point for MU2net, the calculated output is $0.862 \mathrm{~Gy} / 100 \mathrm{MU}$.

\section{Output Factors}

Measured square field output factors were compared to calculated values for MU2net and Monaco for field sizes ranging from $1.0 \times 1.0 \mathrm{~cm}^{2}$ to $22.0 \times 22.0 \mathrm{~cm}^{2}$. Measurements on the Unity were performed as part of the Elekta beam data collection work using a MicroDiamond detector (PTW60019 S/N: 123297) and Semiflex (PTW31010 S/N: 007799) ionization chamber, PTW UNIDOS electrometer (Webline S/N: 00017, firmware v2.06 S/N: 2172) and an Elekta supplied water tank (PTW Beamscan MR S/N: 181208). The MicroDiamond detector was used for fields $\leq 10.0 \times 10.0 \mathrm{~cm}^{2}$ and the Semiflex was used for fields $\geq 5.0 \times 5.0 \mathrm{~cm}^{2}$. Measurement points were positioned on the central axis at a depth of $5.0 \mathrm{~cm}$, SSDs of $133.5 \mathrm{~cm}$ and $138.5 \mathrm{~cm}$. For the $1.0 \times 1.0 \mathrm{~cm}^{2}$ and $2.0 \times 2.0 \mathrm{~cm}^{2}$ fields the relevant field dependent chamber correction factors from TRS483 [12] (0.984 and 0.997 respectively) were applied to the measured data. In addition, EBT3 film was used to determine OFs at both depths for field sizes $\leq 10.0 \times$ $10.0 \mathrm{~cm}^{2}$. To obtain film measurements at the SSDs referred to above PTW RW3 slab phantom stacks $30.0 \mathrm{~cm} \times 30.0 \mathrm{~cm} \times 19.0 \mathrm{~cm}$ and $30.0 \mathrm{~cm} \times 30.0 \mathrm{~cm} \times 14.0 \mathrm{~cm}$ were used, given that the isocentre is 14.0 $\mathrm{cm}$ above the fixed height couch position. The film was exposed to $100 \mathrm{MU}$ with four calibration films 0 MU, $50 \mathrm{MU}, 100 \mathrm{MU}$ and $200 \mathrm{MU}$. The films were scanned with an EPSON Expression 10000XL and analysed using FilmQA ${ }^{\text {TM }}$ Pro (Ashland ISP Advanced Materials, NJ, USA).

\section{Directional dependence}

Due to the non-uniform attenuation of the cryostat there is a corresponding output variation with gantry angle and this was quantified by Elekta as part of their pre-clinical testing (Fig. 2, data courtesy of Elekta). In addition, the transmission and associated attenuation of the treatment beam through the couch and posterior coil (located beneath the couch) is dependent on the gantry angle (for gantry angles $120.0^{\circ}-$ 
$240.0^{\circ}$ the beam transits the couch). The various attenuation processes together with any machine related output fluctuations determine the net variation in output with gantry angle at the isocentre. In order to determine this variation, measurements were performed using a Farmer chamber (PTW30013 S/N: 011298) and PTW UNIDOS electrometer (Webline S/N: 00017), with the chamber centred in a $6.0 \mathrm{~cm}$ diameter water filled cylindrical phantom. Using the EPID the long axis of the cylinder/chamber was aligned parallel to the $y$-axis, $14.0 \mathrm{~cm}$ above the couch, and with the chamber reference point at the isocenter. The cylindrical chamber holder, developed on site, can be rotated in $15.0^{\circ}$ increments and maintains the same orientation with respect to the beam as the gantry is rotated by the same angle. Chamber measurements for $100 \mathrm{MU}$ and a field size of $5.0 \times 5.0 \mathrm{~cm}^{2}$ were recorded every $15.0^{\circ}$ for gantry angles ranging from $0.0^{\circ}$ to $345.0^{\circ}$, avoiding the cryostat pipe at $13.0^{\circ}$, that connects the split superconducting $B_{0}$ coils. Measured output values at each angle were normalized to the $G 90$ value.

A model of the cylindrical phantom (Relative Electron Density $(R E D=1.000)$ was developed in Monaco v5.4 and the dose was determined at the phantom centre for the same field size and range of gantry angles as described above. Monaco calculations include attenuation contributions for the cryostat, couch and posterior coil. For each gantry angle, corresponding Monaco plans were exported to MU2net and dose is calculated. However, it is important to note that the cryostat and posterior coil do not feature in the MU2net computations. There is no cryostat contour included in Monaco and the planning system does not model the radiation transmission through the cryostat. However, cryostat attenuation is accounted for by applying an angular dependent output correction determined by Elekta (Fig. 2). In the current version of Monaco, the posterior imaging coil is not a contoured structure. As such the coil can be visualized and is included in dosimetric calculations ( $R E D=0.181)$ but cannot be exported. To estimate the dosimetric effect of including the posterior coil in the MU2net dose computation, a coil structure mirroring that in Monaco was contoured over the posterior coil. The mirrored structure, layered above the couch structures, was assigned the RED of the posterior coil. This structure, in a non-clinical workflow, was exported to MU2net.

Additionally, the effect of not including the posterior coil in Monaco was investigated. Here the mirrored coil structure was assigned a RED of 0.010 , effectively rendering the coil radiotransparent. This structure was also exported to MU2net. The angular dependence of the central axis dose calculated using MU2net with the mirrored and radiotransparent coil structure was determined.

\section{Custom Fields}

During Elekta data collection a selection central axis square field OFs are measured using the Elekta supplied water tank for the purpose of beam modelling. In addition, measurements for customer defined fields were performed at gantry $0.0^{\circ}$. Referred to as custom fields in this work these fields consisted of rectangular fields $14.0 \times 4.0 \mathrm{~cm}^{2}$ and $4.0 \times 14.0 \mathrm{~cm}^{2}$ and $5.0 \times 5.0 \mathrm{~cm}^{2}$ square fields (Fig. 3. Custom fields $4.0 \times 14.0 \mathrm{~cm}^{2}$ were centred on axis at $X= \pm 24.0 \mathrm{~cm}, \pm 12.0 \mathrm{~cm}$ and $0.0 \mathrm{~cm}$. Similarly, $14.0 \times 4.0$ $\mathrm{cm}^{2}$ were centrally located at $Y= \pm 7.0 \mathrm{~cm}$ and $X=Y=0.0 \mathrm{~cm}$. For each of these fields, measurements were performed at three locations. In addition, $5.0 \times 5.0 \mathrm{~cm}^{2}$ fields were centred $(X, Y)$ at $(-18.0 \mathrm{~cm}, \pm 4.0$ 
$\mathrm{cm})$ and $(18.0 \mathrm{~cm}, \pm 4.0 \mathrm{~cm})$ and $X=Y=0$. These fields were selected to attempt to cover a large area of the maximum collimator opening. For each field, charge readings at $10.0 \mathrm{~cm}$ depth, SSD $=133.5 \mathrm{~cm}, 200$ MU were obtained using a PTW MicroDiamond detector and a PTW UNIDOS electrometer. Readings were normalized to that of a $10.0 \times 10.0 \mathrm{~cm}^{2}$ field to determine output factors.

Monaco DRPs for custom fields were computed using a phantom $(54.5 \mathrm{~cm} \times 30.0 \mathrm{~cm} \times 24.0 \mathrm{~cm}$, forced $R E D=1.000$ ) to match the water tank dimensions during data acquisition. Dose calculations for $200 \mathrm{MU}$, SSD $133.5 \mathrm{~cm}$, gantry angle $0.0^{\circ}$ and depth $10.0 \mathrm{~cm}$ were obtained for each field. The beam exclusion zone included in the current clinical beam model, accounts for a larger pipe and disables dose calculation for a $4.0 \times 14.0 \mathrm{~cm}^{2}$ field at $X=24.0 \mathrm{~cm}$. The beam exclusion zone associated with the magnet cryostat pipe, required the use of an earlier Elekta beam model that enabled point doses for the $4.0 \times 14.0 \mathrm{~cm}^{2}, \mathrm{X}=$ $24.0 \mathrm{~cm}$ field to be calculated.

\section{Sample Elekta Plans - Dose Reference Point evaluation}

As part of the Elekta beam validation process, nine step and shoot IMRT plans were calculated in Monaco on a phantom $(30.0 \mathrm{~cm} \times 30.0 \mathrm{~cm} \times 19.0 \mathrm{~cm}$, forced RED $=1.000)$ As an independent verification, each of the IMRT plans were delivered on EBT3 film and compared to Monaco dose maps using gamma analysis with criteria of $2.0 \%$ global dose difference and $2.0 \mathrm{~mm}$ distance-to-agreement (DTA) where $\geq 95.0 \%$ of points with a gamma $<1.0$ was considered a pass. These plans were also used to compare Monaco and MU2net calculated dose for selected DRPs. Each DRP was calculated with either all gantry angles set to zero (perpendicularly) or with planned treatment angles (compositely) using a $30.0 \mathrm{~cm} \times 30.0 \mathrm{~cm} \times 17.8$ $\mathrm{cm}$ phantom, (forced RED $=1.000$ ). The Monaco dose for each plan was calculated with a grid spacing of $0.2 \mathrm{~cm}$ and a statistical uncertainty of $3.0 \%$ per control point. Each plan with phantom and couch structures was exported to MU2net and dose to the DRPs for each beam were computed.

\section{Clinical Cases}

\section{Pretreatment QA}

During pretreatment QA, the reference plan from which adapted plans are created is verified. For this QA, fraction zero, a DRP is assigned to each beam in Monaco and dose is calculated. Broadly speaking DRPs are selected in a uniform dose region within the planning target volume (PTV). The DICOM RT Plan and RT StructureSet (couch and patient) are exported to MU2net for secondary dose computation. MU2net DRP values and corresponding Monaco mean dose DRP values are copied and manually compared using excel. When MU2net versus Monaco DRP discrepancies were greater than $\pm 5.0 \%$, MU2net DRPs were reselected to achieve discrepancies within this range. In some cases, this could not be achieved and the DRP discrepancy was then taken as a baseline for that beam during the adaptive planning process. For such beams $\pm 5.0 \%$ discrepancies from the baseline value were used during the online independent MU checking procedure. For clinical workflow purposes average MU2net calculation times were approximately 1.0 minute. On average the treatment time per patient was 45.0 minutes. 


\section{Treatment}

Our first patient on the Elekta Unity was treated in December 2019 and a total of 215 fractions (1552 beams) were delivered in 2020. Some of the more common treatment sites included: intact prostate, common iliac node, external iliac node, pelvic node, para-aortic node and seminal vesicles. The PTVs of these treatment sites ranged from $1.9 \mathrm{~cm}^{3}$ to $162.4 \mathrm{~cm}^{3}$, with field sizes varying from approximately $1.0 \times$ $1.0 \mathrm{~cm}^{2}$ to $8.0 \times 8.0 \mathrm{~cm}^{2}$. To observe if PTV size has an effect on the Monaco versus MU2net percentage differences, the patient data was separated into two categories: prostate (PTV > 77.0 $\mathrm{cm}^{3}$ ) and "nodes/vesicles" (PTV $<21.0 \mathrm{~cm}^{3}$ ).

\section{Results}

\section{Output factors}

The variation of measured and calculated OFs for the field sizes and SSDs referred to above are shown in Fig. 4 and Fig. 5. Measured and Monaco OFs for both SSDs agreed within $1.0 \%$ for all field sizes.

At SSD $133.5 \mathrm{~cm}$ measured and MU2net OFs, for field sizes $1.0 \times 1.0 \mathrm{~cm}^{2}$ to $14.0 \times 14.0 \mathrm{~cm}^{2}$ are within \pm $1.7 \%$ and as the field size increases from $15.0 \times 15.0 \mathrm{~cm}^{2}$ to $22.0 \times 22.0 \mathrm{~cm}^{2}$ the discrepancy ranges from $2.1 \%$ to $3.5 \%$. At SSD $138.5 \mathrm{~cm}$ measured and MU2net OFs, for field sizes $1.0 \times 1.0 \mathrm{~cm}^{2}$ to $15.0 \times$ $15.0 \mathrm{~cm}^{2}$ are within $\pm 2.2 \%$ and for the larger field sizes $20.0 \times 20.0 \mathrm{~cm}^{2}$ to $22.0 \times 22.0 \mathrm{~cm}^{2}$ the discrepancy increases to $4.1 \%$.

For $133.5 \mathrm{~cm}$ and $138.5 \mathrm{~cm} \mathrm{SSD}$, measured and EBT3 OFs for field sizes $3.0 \times 3.0 \mathrm{~cm}^{2}$ to $10.0 \times 10.0 \mathrm{~cm}^{2}$ were within $\pm 1.4 \%$ and $\pm 2.2 \%$ respectively and for field sizes below $2.0 \times 2.0 \mathrm{~cm}^{2}$ the maximum discrepancies are $-2.7 \%$ and $-2.9 \%$ respectively.

\section{Directional dependence}

In the absence of the couch and posterior coil structures the angular dependence of the measured output is shown in Fig. 2. Angular dependent normalised (G90) output values for Monaco, MU2net (with/without posterior coil) and measured data are shown in Fig. 6 and presented in Table 1. Percentage differences for both Monaco and MU2net relative to measured data are shown in parentheses. In the current clinical workflow, MU2net computations do not include the posterior coil since the RT StructureSet exported from Monaco does not include this structure as discussed above. Absolute percentage differences between MU2net and measured data vary from $1.3 \%$ to $5.3 \%$ (mean $4.2 \%$ ) at gantry angles ranging from $120.0^{\circ}$ to $240.0^{\circ}$. With the inclusion of a posterior coil in MU2net absolute the mean difference decreases to 2.4 $\%$, differences at gantry angles of $120.0^{\circ}$ and $240.0^{\circ}$ are unchanged. With or without the posterior coil absolute differences between MU2net and measured data vary by $-0.9 \%$ to $1.7 \%$ for gantry angles $0.0^{\circ}$ to $105.0^{\circ}$ and $250.0^{\circ}$ to $355.0^{\circ}$. For the same range of angles, percentage differences between Monaco and measured data are within $\pm 0.2 \%$. However, in the range of $120.0^{\circ}$ to $240.0^{\circ}$ Monaco output values are 
less than measured values with percentage differences in the range of $-1.1 \%$ to $-3.1 \%$ with a mean difference $-1.9 \%$.

Table 1 Normalized output values for measured, Monaco and MU2net (with/without posterior coil) with values in parentheses showing percentage differences relative to measured data. For gantry angles $120.0^{\circ}-240.0^{\circ}$ the beam transits the couch 


\begin{tabular}{|c|c|c|c|c|c|c|c|}
\hline \multirow{2}{*}{ 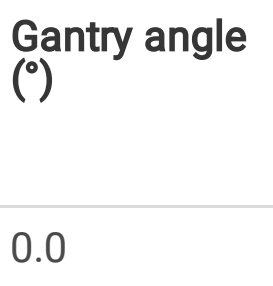 } & \multirow{2}{*}{$\begin{array}{l}\text { Measured } \\
\text { output }\end{array}$} & \multicolumn{2}{|c|}{$\begin{array}{l}\text { Monaco } \\
\text { output }\end{array}$} & \multicolumn{2}{|c|}{$\begin{array}{l}\text { MU2net } \\
\text { output } \\
\text { no coil }\end{array}$} & \multicolumn{2}{|c|}{$\begin{array}{l}\text { MU2net output posterior } \\
\text { coil }\end{array}$} \\
\hline & & 1.01 & $(0.2)$ & 1.00 & $(-0.2)$ & 1.00 & $(-0.2)$ \\
\hline \multicolumn{8}{|l|}{15.0} \\
\hline 30.0 & 1.01 & 1.01 & $(0.1)$ & 1.00 & $(-0.9)$ & 1.00 & $(-0.9)$ \\
\hline 45.0 & 1.00 & 1.00 & $(0.2)$ & 1.00 & $(0.4)$ & 1.00 & $(0.4)$ \\
\hline 60.0 & 1.00 & 1.00 & $(0.0)$ & 1.00 & $(0.1)$ & 1.00 & $(0.1)$ \\
\hline 75.0 & 0.99 & 0.99 & $(0.0)$ & 1.00 & $(0.9)$ & 1.00 & $(0.9)$ \\
\hline 90.0 & 1.00 & 1.00 & $(0.0)$ & 1.00 & $(0.0)$ & 1.00 & $(0.0)$ \\
\hline 105.0 & 0.99 & 0.99 & $(0.1)$ & 1.00 & (1.3) & 1.00 & (1.3) \\
\hline 120.0 & 0.81 & 0.78 & $(-3.1)$ & 0.82 & (1.3) & 0.82 & (1.3) \\
\hline 135.0 & 0.83 & 0.81 & $(-1.9)$ & 0.87 & $(4.9)$ & 0.85 & (2.5) \\
\hline 150.0 & 0.86 & 0.85 & $(-1.1)$ & 0.89 & (4.4) & 0.87 & $(1.8)$ \\
\hline 165.0 & 0.88 & 0.86 & $(-1.8)$ & 0.91 & (3.5) & 0.89 & (1.4) \\
\hline 180.0 & 0.88 & 0.86 & $(-2.3)$ & 0.91 & (4.3) & 0.90 & $(2.2)$ \\
\hline 195.0 & 0.88 & 0.85 & $(-2.7)$ & 0.91 & $(4.0)$ & 0.89 & $(1.8)$ \\
\hline 210.0 & 0.86 & 0.84 & $(-1.5)$ & 0.90 & (5.3) & 0.88 & (2.8) \\
\hline 225.0 & 0.82 & 0.81 & $(-1.4)$ & 0.86 & (5.3) & 0.84 & $(2.8)$ \\
\hline 240.0 & 0.78 & 0.77 & $(-1.1)$ & 0.81 & (4.9) & 0.81 & $(4.9)$ \\
\hline 255.0 & 0.98 & 0.98 & $(-0.0)$ & 1.00 & (1.6) & 1.00 & $(1.6)$ \\
\hline 270.0 & 0.98 & 0.98 & $(-0.0)$ & 1.00 & $(1.8)$ & 1.00 & $(1.8)$ \\
\hline 285.0 & 0.98 & 0.98 & $(0.0)$ & 1.00 & (1.7) & 1.00 & (1.7) \\
\hline 300.0 & 0.99 & 0.98 & $(-0.0)$ & 1.00 & (1.5) & 1.00 & $(1.5)$ \\
\hline 315.0 & 0.99 & 0.99 & $(0.0)$ & 1.00 & (1.5) & 1.00 & $(1.5)$ \\
\hline 330.0 & 1.00 & 1.00 & $(0.2)$ & 1.00 & $(-0.1)$ & 1.00 & $(-0.1)$ \\
\hline 345.0 & 1.00 & 1.00 & $(-0.1)$ & 1.00 & $(-0.1)$ & 1.00 & $(-0.1)$ \\
\hline
\end{tabular}


Measured, MU2net and Monaco custom field OFs are shown in Table 2, percentage differences relative to measured dose are shown in parentheses. Table 2.a displays the OFs for a $4.0 \times 14.0 \mathrm{~cm}^{2}$ field which were shifted off axis in the $\pm X$ directions. At a field offset of $X=-24.0 \mathrm{~cm}$ the average percentage difference between the measured and MU2net OFs is $8.4 \%$ whereas for $X=24.0 \mathrm{~cm}$ the difference is 11.6 $\%$. As the offset reduces to $X= \pm 12.0 \mathrm{~cm}$ the average differences reduce to $2.8 \%(-12.0 \mathrm{~cm})$ and $5.3 \%$ $(12.0 \mathrm{~cm})$. On central axis the average percentage difference is $0.5 \%$. Clearly as the offset is reduced for either direction, differences decrease and are larger in the positive $\mathrm{X}$ direction.

Measured and Monaco OF discrepancies in the - $\mathrm{X}$ direction have a larger absolute average percentage difference $(-4.1 \%$ at $-24.0 \mathrm{~cm}$ and $-1.9 \%$ at $-12.0 \mathrm{~cm})$ compared to differences in the X direction $(-1.9 \%$ at $24.0 \mathrm{~cm}$ and $0.9 \%$ at $-12 \mathrm{~cm}$ ). On CAX the average percentage difference is $0.6 \%$.

The OFs for the $14.0 \times 4.0 \mathrm{~cm}^{2}$ field shifted in the $\pm Y$ directions are shown in Table 2.b. Average percentage differences between measured and MU2net OFs in the $\pm Y$ directions and on CAX are $1.1 \%$ and $-0.6 \%$ respectively. Similarly, for measured and Monaco OFs, the average percentage differences are $0.3 \%$ in the $\pm Y$ directions and $0.5 \%$ on CAX.

OFs for $5.0 \times 5.0 \mathrm{~cm}^{2}$ square fields $\pm 18.0 \mathrm{~cm}$ off axis in the $X$ direction and $\pm 4.0 \mathrm{~cm}$ off axis in the $Y$ direction are shown in Table 2.c. Following the trend in Table 2.a, the average percentage difference between measured and MU2net for the fields at $X=+18.0 \mathrm{~cm}$ and $X=-18 \mathrm{~cm}$ off axis is $8.9 \%$ and $6.6 \%$ respectively. On CAX the percentage difference is $-1.2 \%$. For measured and Monaco OFs, the average percentage difference for the off-axis field in the $-X$ direction is $-2.2 \%$, and $1.2 \%$ in the $+X$ direction. On CAX the percentage difference is $0.1 \%$.

Table 2 Custom field OFs for measured, MU2net and Monaco. (a) $4.0 \times 14.0 \mathrm{~cm}^{2}$ field (X offsets of - 24.0 $\mathrm{cm},-12.0 \mathrm{~cm}, 12.0 \mathrm{~cm}, 24.0 \mathrm{~cm}$ and on CAX), (b) $14 \times 4 \mathrm{~cm}^{2}$ field ( $Y$ offset of $-7 \mathrm{~cm}, 7 \mathrm{~cm}$ and on CAX) and (c) $5.0 \times 5.0 \mathrm{~cm}^{2}$ field (X offset of $-18 \mathrm{~cm}, 18 \mathrm{~cm}$ and on CAX). Percentage differences in parentheses are relative to measured data 


\section{(a) Field Size $4.0 \times 14.0 \mathrm{~cm}^{2}$}

\begin{tabular}{|c|c|c|c|c|c|c|c|c|}
\hline \multicolumn{2}{|c|}{ Field Offset } & \multicolumn{2}{|c|}{ Measurement Point Offset } & \multirow[t]{2}{*}{ OF Measured } & \multirow{2}{*}{\multicolumn{2}{|c|}{$\begin{array}{l}\text { OF } \\
\text { MU2net }\end{array}$}} & \multirow{2}{*}{\multicolumn{2}{|c|}{$\begin{array}{l}\text { OF } \\
\text { Monaco }\end{array}$}} \\
\hline \multirow{2}{*}{$\begin{array}{l}X \\
-24.0\end{array}$} & \multirow{2}{*}{$\begin{array}{l}\mathbf{Y} \\
0.0\end{array}$} & \multirow{2}{*}{$\begin{array}{l}X \\
-24.0\end{array}$} & $\mathbf{Y}$ & & & & & \\
\hline & & & 0.0 & 0.45 & 0.48 & (8.6) & 0.43 & $(-4.2)$ \\
\hline & & -24.0 & 5.0 & 0.43 & 0.47 & (8.4) & 0.41 & $(-4.3)$ \\
\hline & & -24.0 & -5.0 & 0.43 & 0.47 & (8.3) & 0.41 & $(-3.8)$ \\
\hline \multirow[t]{3}{*}{24.0} & 0.0 & 24.0 & 0.0 & 0.44 & 0.49 & $(11.7)$ & 0.43 & $(-1.2)$ \\
\hline & & 24.0 & 5.0 & 0.42 & 0.47 & (11.7) & 0.42 & $(-1.1)$ \\
\hline & & 24.0 & -5.0 & 0.42 & 0.47 & $(11.3)$ & 0.42 & $(-1.3)$ \\
\hline \multirow[t]{3}{*}{-12.0} & 0.0 & -12.0 & 0.0 & 0.69 & 0.71 & $(2.8)$ & 0.68 & $(-2.2)$ \\
\hline & & -12.0 & 5.0 & 0.66 & 0.68 & (2.8) & 0.65 & $(-1.7)$ \\
\hline & & -12.0 & -5.0 & 0.66 & 0.68 & $(2.8)$ & 0.65 & $(-1.8)$ \\
\hline \multirow[t]{3}{*}{12.0} & 0.0 & 12.0 & 0.0 & 0.68 & 0.71 & $(4.6)$ & 0.69 & (1.1) \\
\hline & & 12.0 & 5.0 & 0.65 & 0.69 & (6.8) & 0.66 & $(0.9)$ \\
\hline & & 12.0 & -5.0 & 0.65 & 0.68 & (4.4) & 0.66 & $(0.8)$ \\
\hline \multirow[t]{3}{*}{0.0} & 0.0 & 0.0 & 0.0 & 0.94 & 0.93 & $(-1.0)$ & 0.95 & $(0.6)$ \\
\hline & & 0.0 & 5.0 & 0.86 & 0.89 & (2.8) & 0.86 & $(0.0)$ \\
\hline & & 0.0 & -5.0 & 0.86 & 0.85 & $(-0.3)$ & 0.87 & $(1.2)$ \\
\hline
\end{tabular}

(b) Field Size $14.0 \times 4.0 \mathrm{~cm}^{2}$

\section{Field Offset Measurement Point Offset OF Measured OF OF}

\begin{tabular}{|c|c|c|c|c|c|c|c|c|}
\hline \multirow{2}{*}{$\begin{array}{l}X \\
0.0\end{array}$} & \multirow{2}{*}{$\begin{array}{l}Y \\
-7.0\end{array}$} & \multirow{2}{*}{$\begin{array}{l}X \\
0.0\end{array}$} & \multicolumn{2}{|l|}{ Y } & \multicolumn{2}{|c|}{ MU2net } & \multicolumn{2}{|c|}{ Monaco } \\
\hline & & & -7.0 & 0.81 & 0.83 & (1.4) & 0.82 & (0.6) \\
\hline & & 5.0 & -7.0 & 0.76 & 0.77 & (1.8) & 0.77 & (1.8) \\
\hline & & -5.0 & -7.0 & 0.77 & 0.77 & (1.1) & 0.76 & $(-0.7)$ \\
\hline \multirow[t]{3}{*}{0.0} & 7.0 & 0.0 & 7.0 & 0.82 & 0.82 & $(0.7)$ & 0.82 & $(0.1)$ \\
\hline & & 5.0 & 7.0 & 0.76 & 0.77 & (1.2) & 0.77 & (1.5) \\
\hline & & -5.0 & 7.0 & 0.77 & 0.77 & $(0.6)$ & 0.76 & $(-1.3)$ \\
\hline \multirow[t]{2}{*}{0.0} & 0.0 & 0.0 & 0.0 & 0.94 & 0.93 & $(-0.9)$ & 0.95 & (0.6) \\
\hline & & 5.0 & 0.0 & 0.86 & 0.85 & $(-0.2)$ & 0.87 & (1.6) \\
\hline
\end{tabular}




\begin{tabular}{|c|c|c|c|c|c|c|c|c|}
\hline \multirow{2}{*}{\multicolumn{4}{|c|}{$\begin{array}{c}-5.0 \\
\text { (c) Field Size } 5.0 \times 5.0 \mathrm{~cm}^{2}\end{array}$}} & \multirow[t]{2}{*}{0.86} & \multirow{2}{*}{0.86} & \multirow{2}{*}{$(-0.8)$} & \multirow{2}{*}{0.86} & \multirow{2}{*}{$(-0.7)$} \\
\hline & & & & & & & & \\
\hline \multicolumn{2}{|c|}{ Field Offset } & \multicolumn{2}{|c|}{ Measurement Point Offset } & \multirow[t]{2}{*}{ OF Measured } & \multirow{2}{*}{\multicolumn{2}{|c|}{$\begin{array}{l}\text { OF } \\
\text { MU2net }\end{array}$}} & \multirow{2}{*}{\multicolumn{2}{|c|}{$\begin{array}{l}\text { OF } \\
\text { Monaco }\end{array}$}} \\
\hline$x$ & $\mathrm{Y}$ & $\mathbf{X}$ & $\mathrm{Y}$ & & & & & \\
\hline-18.0 & 4.0 & -18.0 & 4.0 & 0.53 & 0.57 & $(6.6)$ & 0.52 & $(-2.3)$ \\
\hline-18.0 & -4.0 & -18.0 & -4.0 & 0.53 & 0.57 & $(6.6)$ & 0.52 & $(-2.1)$ \\
\hline 18.0 & 4.0 & 18.0 & 4.0 & 0.52 & 0.57 & $(8.9)$ & 0.53 & $(1.2)$ \\
\hline 18.0 & -4.0 & 18.0 & -4.0 & 0.52 & 0.57 & (8.8) & 0.53 & $(1.2)$ \\
\hline 0.0 & 0.0 & 0.0 & 0.0 & 0.92 & 0.91 & $(-1.2)$ & 0.92 & $(-0.1)$ \\
\hline
\end{tabular}

\section{Sample IMRT Plans}

With individual beams calculated perpendicularly from gantry zero, plan specific percentage point dose differences for MU2net and Monaco are shown Table 3. It is observed that with a DRP selected for each beam, all differences between Monaco and MU2net DRPs are less than $\pm 5.0 \%$.

Point dose comparisons for all IMRT plans with their beams calculated at planned treatment angles are shown in Table 4. With DRPs selected per beam, Monaco and MU2net agree within $\pm 5.0 \%$ for all plans except for beam angle G132 of plan Prostate 1 (6.1\% discrepancy). Even after reselection of this DRP the discrepancy did not improve.

Table 3 IMRT plans collapsed to zero beam angles: Percentage differences of Monaco and MU2net point dose DRP comparison for each individual beam 


\begin{tabular}{|llllllllllll|}
\hline Site & \multicolumn{2}{l}{ Beam \# } & & & & & & & & & \\
& $\mathbf{1}$ & $\mathbf{2}$ & $\mathbf{3}$ & $\mathbf{4}$ & $\mathbf{5}$ & $\mathbf{6}$ & $\mathbf{7}$ & $\mathbf{8}$ & $\mathbf{9}$ & $\mathbf{1 0}$ & $\mathbf{1 1}$ \\
\hline 11 field & -1.7 & 0.2 & 2.9 & 1.6 & 1.2 & 4.1 & -1.0 & -2.9 & -2.7 & 0.8 & 1.4 \\
\hline Head \& Neck & -0.1 & -0.5 & 3.9 & 2.5 & 2.9 & -1.0 & 3.9 & 0.3 & -1.2 & & \\
\hline Prostate 9 & -0.6 & 0.5 & 4.2 & 0.3 & 0.5 & 0.2 & -0.1 & -0.2 & 1.2 & & \\
\hline Multitarget & 0.5 & 0.7 & 2.2 & -0.4 & 0.5 & 3.2 & -1.0 & & & & \\
\hline Prostate 1 & 1.5 & 2.0 & -0.6 & 2.4 & 0.5 & -1.6 & -1.7 & & & \\
\hline Prostate 2 & 0.9 & 3.7 & 0.1 & 4.2 & 0.4 & 2.1 & 2.5 & & & & \\
\hline Prostate 7 & -2.7 & 3.0 & 0.9 & 2.4 & -2.5 & -2.8 & 0.7 & & & & \\
\hline Abdomen & 3.0 & 1.9 & 2.1 & -0.9 & 1.1 & -0.2 & & & & & \\
\hline Lung & 2.0 & 4.3 & 1.2 & 1.6 & 0.0 & & & & & & \\
\hline
\end{tabular}

Table 4 IMRT plans with planned beam angles: Percentage differences for Monaco and MU2net point dose DRPs for individual beams as well as average percentage differences (Avg Diff) for all beams of each plan \pm 1.0 standard deviation 


\begin{tabular}{|c|c|c|c|c|c|c|c|c|c|c|c|c|}
\hline \multirow{3}{*}{$\begin{array}{l}\text { Site } \\
11 \text { Field }\end{array}$} & \multicolumn{11}{|c|}{ Planned beam angles } & \multirow{3}{*}{$\begin{array}{l}\text { Avg } \\
\text { Diff (\% } \\
\pm \text { SD) } \\
0.4 \pm \\
2.4\end{array}$} \\
\hline & $\begin{array}{l}\text { G } \\
162\end{array}$ & $\begin{array}{l}G \\
130\end{array}$ & $\begin{array}{l}\text { G } \\
97\end{array}$ & $\begin{array}{l}G \\
65\end{array}$ & $\begin{array}{l}\text { G } \\
32\end{array}$ & G 0 & $\begin{array}{l}\text { G } \\
324\end{array}$ & $\begin{array}{l}\text { G } \\
292\end{array}$ & $\begin{array}{l}G \\
259\end{array}$ & $\begin{array}{l}\text { G } \\
227\end{array}$ & $\begin{array}{l}\text { G } \\
195\end{array}$ & \\
\hline & 1.7 & 1.2 & 2.9 & -3.5 & 0.4 & -3.9 & 2.5 & -2.0 & 1.0 & 2.5 & 1.6 & \\
\hline \multirow[t]{2}{*}{$\begin{array}{l}\text { Head \& } \\
\text { Neck }\end{array}$} & $\begin{array}{l}G \\
160\end{array}$ & $\begin{array}{l}G \\
120\end{array}$ & $\begin{array}{l}G \\
80\end{array}$ & $\begin{array}{l}G \\
40\end{array}$ & G 0 & $\begin{array}{l}G \\
320\end{array}$ & $\begin{array}{l}G \\
280\end{array}$ & $\begin{array}{l}G \\
240\end{array}$ & $\begin{array}{l}G \\
200\end{array}$ & & & \multirow[t]{2}{*}{$\begin{array}{l}0.4 \pm \\
3.1\end{array}$} \\
\hline & 3.3 & 4.4 & 1.4 & -2.3 & 0.1 & -1.4 & -4.8 & -1.0 & 4.0 & & & \\
\hline \multirow[t]{2}{*}{ Prostate 9} & $\begin{array}{l}G \\
160\end{array}$ & $\begin{array}{l}G \\
120\end{array}$ & $\begin{array}{l}G \\
80\end{array}$ & $\begin{array}{l}G \\
40\end{array}$ & G 0 & $\begin{array}{l}G \\
320\end{array}$ & $\begin{array}{l}G \\
280\end{array}$ & $\begin{array}{l}G \\
240\end{array}$ & $\begin{array}{l}G \\
200\end{array}$ & & & \multirow[t]{2}{*}{$\begin{array}{l}0.6 \pm \\
2.8\end{array}$} \\
\hline & 1.5 & -2.1 & 4.3 & 3.0 & 2.2 & -0.7 & -1.4 & -4.0 & 2.7 & & & \\
\hline \multirow[t]{2}{*}{ Multitarget } & $\begin{array}{l}G \\
153\end{array}$ & $\begin{array}{l}\text { G } \\
102\end{array}$ & $\begin{array}{l}\text { G } \\
51\end{array}$ & G 0 & $\begin{array}{l}G \\
306\end{array}$ & $\begin{array}{l}G \\
255\end{array}$ & $\begin{array}{l}\text { G } \\
204\end{array}$ & & & & & \multirow[t]{2}{*}{$\begin{array}{l}-0.2 \pm \\
3.4\end{array}$} \\
\hline & 4.3 & -0.4 & -4.6 & -1.1 & -4.1 & 1.7 & 2.8 & & & & & \\
\hline \multirow[t]{2}{*}{ Prostate 1} & $\begin{array}{l}G \\
183\end{array}$ & $\begin{array}{l}G \\
132\end{array}$ & $\begin{array}{l}\text { G } \\
81\end{array}$ & $\begin{array}{l}\text { G } \\
30\end{array}$ & $\begin{array}{l}G \\
336\end{array}$ & $\begin{array}{l}G \\
285\end{array}$ & $\begin{array}{l}\text { G } \\
234\end{array}$ & & & & & \multirow[t]{2}{*}{$\begin{array}{l}2.0 \pm \\
4.2\end{array}$} \\
\hline & 2.1 & 6.4 & 4.6 & -3.6 & 3.5 & 4.9 & -4.0 & & & & & \\
\hline \multirow[t]{2}{*}{ Prostate 2} & $\begin{array}{l}G \\
153\end{array}$ & $\begin{array}{l}\text { G } \\
102\end{array}$ & $\begin{array}{l}\text { G } \\
51\end{array}$ & G 0 & $\begin{array}{l}\text { G } \\
306\end{array}$ & $\begin{array}{l}\text { G } \\
255\end{array}$ & $\begin{array}{l}\text { G } \\
204\end{array}$ & & & & & \multirow[t]{2}{*}{$\begin{array}{l}1.1 \pm \\
2.4\end{array}$} \\
\hline & 4.7 & 1.0 & 0.8 & 2.2 & -3.4 & 1.1 & 1.7 & & & & & \\
\hline \multirow[t]{2}{*}{ Prostate 7} & $\begin{array}{l}\text { G } \\
154\end{array}$ & $\begin{array}{l}\text { G } \\
102\end{array}$ & $\begin{array}{l}\mathbf{G} \\
50\end{array}$ & G 0 & $\begin{array}{l}\text { G } \\
310\end{array}$ & $\begin{array}{l}\text { G } \\
258\end{array}$ & $\begin{array}{l}\text { G } \\
206\end{array}$ & & & & & \multirow[t]{2}{*}{$\begin{array}{l}0.9 \pm \\
1.6\end{array}$} \\
\hline & 0.5 & 3.7 & -0.4 & 0.5 & -0.6 & 0.3 & 2.4 & & & & & \\
\hline \multirow[t]{2}{*}{ Abdomen } & $\begin{array}{l}G \\
165\end{array}$ & $\begin{array}{l}\text { G } \\
105\end{array}$ & $\begin{array}{l}G \\
60\end{array}$ & $\begin{array}{l}G \\
30\end{array}$ & G 0 & $\begin{array}{l}G \\
320\end{array}$ & & & & & & \multirow[t]{2}{*}{$\begin{array}{l}1.7 \pm \\
2.0\end{array}$} \\
\hline & 3.8 & 1.2 & 4.3 & -1.2 & 1.5 & 0.8 & & & & & & \\
\hline \multirow[t]{2}{*}{ Lung } & $\begin{array}{l}\text { G } \\
195\end{array}$ & $\begin{array}{l}\mathbf{G} \\
35\end{array}$ & G 0 & $\begin{array}{l}\text { G } \\
300\end{array}$ & $\begin{array}{l}\text { G } \\
246\end{array}$ & & & & & & & \multirow[t]{2}{*}{$\begin{array}{l}3.2 \pm \\
1.3\end{array}$} \\
\hline & 3.5 & 4.0 & 1.0 & 3.1 & 4.2 & & & & & & & \\
\hline
\end{tabular}

\section{Clinical Cases}

A summary of the percentage differences between MU2net and Monaco DRPs bins for each of 1552 treatment beam is displayed in Fig. 8. The beams are split into two categories: prostates (895 beams) 
and nodes/vesicles (657 beams). MU2net point doses tend to be greater than Monaco for the same DRP. Overall, for the prostate fields (average segment size is $28.87 \mathrm{~cm}^{2}$ ) and nodal fields (average segment size is $7.09 \mathrm{~cm}^{2}$ )DRP discrepancies within $\pm 5.0 \%$ for the prostate (average segment size is $28.87 \mathrm{~cm}^{2}$ ) and nodal (average segment size is $7.09 \mathrm{~cm}^{2}$ ) beams are $84.7 \%$ and $73.8 \%$, respectively. In addition, the average segment area for each beam was recorded and correlated with the Monaco versus MU2net percentage differences, to see if any field size dependence was evident. The relation between the DRP \% discrepancies bins and their respective segment area for each beam, as well as the number of beams in each range, is summarized in Table 5. Even though the standard deviation is relatively large, it is relatively consistent for each site. For the nodes/vesicles the segment area is smaller compared to the prostate cases and most points still pass within $\pm 5.0 \%$.

Table 5 Average segment area for each \% difference range for Monaco versus MU2net, \pm 1 standard deviation as well as the number of beams in each range

\begin{tabular}{|llllllll|}
\hline $\begin{array}{l}\text { Difference } \\
(\%)\end{array}$ & \begin{tabular}{l} 
Prostates \\
\cline { 2 - 7 }
\end{tabular} & $\begin{array}{l}\text { Avg Segment Size } \\
\left(\mathbf{c m}^{2}\right)\end{array}$ & SD & $\begin{array}{l}\text { \# of } \\
\text { beams }\end{array}$ & $\begin{array}{l}\text { Avg Segment Size } \\
\left(\mathbf{c m}^{2}\right)\end{array}$ & SD & $\begin{array}{l}\text { \# of } \\
\text { beams }\end{array}$ \\
\hline$(\infty,-5]$ & - & - & - & 4.16 & 1.91 & 15 \\
\hline$(-5,5)$ & 29.24 & 7.05 & 766 & 7.39 & 3.19 & 568 \\
\hline$[5,6)$ & 29.46 & 6.63 & 58 & 6.39 & 2.62 & 29 \\
\hline$[6,7)$ & 25.39 & 8.06 & 39 & 5.60 & 2.39 & 20 \\
\hline$[7,8)$ & 24.87 & 6.53 & 16 & 6.02 & 2.06 & 13 \\
\hline$[8,9)$ & 22.27 & 6.05 & 8 & 6.20 & 2.94 & 13 \\
\hline$[9,10)$ & 21.27 & 4.51 & 6 & 3.44 & 0.70 & 4 \\
\hline$[10, \infty)$ & 23.11 & 5.50 & 2 & 3.77 & 0.99 & 10 \\
\hline
\end{tabular}

\section{Discussion}

For the on-axis field sizes $\leq 15.0 \times 15.0 \mathrm{~cm}^{2}$, the agreement between MU2net and measured data is acceptable. However, it appears that MU2net is not suitable for calculating output factors for larger field sizes.

The cryostat characterisation (Fig. 2) exhibits approximately a $3.0 \%$ maximum variation in output at the isocentre. In addition to the cryostat, the treatment couch and posterior coil contribute to the overall beam attenuation, Fig. 6 shows that for gantry angles of $0.0^{\circ}, 30.0^{\circ}-105.0^{\circ}$ and $255.0^{\circ}-345.0^{\circ}$, measured and Monaco normalised output values for a $5.0 \times 5.0 \mathrm{~cm}^{2}$ field are in good agreement. For these angles the beam does not traverse the couch or posterior coil. In the range $120.0^{\circ}$ to $240.0^{\circ}$ discrepancies between 
measured and Monaco are observed, with Monaco output consistently lower than measured data. The differences maximum is $3.3 \%$ at $120.0^{\circ}$ and the minimum is $1.4 \%$ at $240.0^{\circ}$. This suggests that the current modelling of the couch and posterior coil in Monaco overestimates the combined attenuation. At present MU2net dose computation incorporates couch attenuation but does not account for cryostat and posterior coil attenuation. Hence for gantry angles $0.0^{\circ}-105.0^{\circ}$ and $255.0^{\circ}-345.0^{\circ}$ differences between MU2net and measured data (Fig. 2) are associated with lack of cryostat modelling. Discrepancies at angles $120.0^{\circ}-240.0^{\circ}$ are potentially associated with deficiencies in the modelling of all sources of beam attenuation.

Figure 8, displays absolute percentage differences between Monaco, Monaco with a radiotransparent posterior coil, MU2net and MU2net + mirrored posterior coil with measured data. Average differences between MU2net and measured data are decreased from 2.1-1.4\% with the mirrored coil model included. The inclusion of a cryostat model would potentially further reduce the differences between MU2net and measured data. In addition, the average discrepancy for Monaco and measured data is $-0.1 \%$ without "the coil" (RED $=0.010)$ compared to $-1.9 \%$ with the coil. Due to the lateral extent of the coil model there is no change in the discrepancy between MU2net and measured output at $120.0^{\circ}$ and $240.0^{\circ}$.

Comparison of measured and Monaco OFs, shows that the off-axis point doses in the - $\mathrm{X}$ direction have a greater discrepancy compared to corresponding fields in the $\mathrm{X}$ direction. However, the opposite is the case when comparing measured with MU2net OFs. For off axis fields at $12.0 \mathrm{~cm}$ and $24.0 \mathrm{~cm}$ in either $\mathrm{X}$ direction the discrepancy between MU2net and measured OFs is within the range $3.0 \%$ to $12.0 \%$. These larger discrepancies are unclear; however, they could be attributed to the limitation of the Clarkson algorithm for off-axis fields as well as the use of $B_{0}=0$ T profiles used in MU2net.

Most DRP discrepancies for Monaco and MU2net were within $\pm 5.0 \%$ for SSIMRT beams delivered at planned gantry angles. An exception to this was the discrepancy of $6.4 \%$ observed for the $\mathrm{G} 132$ beam of plan Prostate 1. The independent verification with EBT3 film returned an agreement $>95.0 \%$ for the above-mentioned gamma criteria. It is noted that this beam traverses the densest part of the couch and the beam enters the solid water phantom at the corner of the stack. A systematic output study of measured, Monaco and MU2net square fields $\leq 5.0 \mathrm{~cm}^{2}$ traversing the couch posteriorly is warranted but outside the scope of this work.

Overall, for all 1552 fractions delivered, approximately $80.0 \%$ of Monaco versus MU2net DRP discrepancies are within $\pm 5.0 \%$. Therefore, discrepancies for prostate beams are on average less than that for nodes/vesicles and this in part is due to segment area and the ability to select DRPs in regions of uniform dose. Given the frequency of MU2net-Monaco discrepancies $> \pm 5.0 \%$ observed in this work, the suitability of MU2net as a standalone independent dose check must be considered within such limitations. Following the reference plan and the identification of baseline discrepancies for the DRPs for all beams, our procedure is to complete plan QA using either EBT3 (maximum dose < 10.0 Gy) or EBT XD (maximum dose > $10.0 \mathrm{~Gy}$ ). Using FilmQA Pro (Ashland ISP Advanced Materials, NJ, USA) and triple channel dosimetry [13], gamma criteria of $2 \% / 2 \mathrm{~mm}$ (global) are applied to each film-Monaco planar dose 
comparison. To date gamma values pass rates $>95 \%$ are achieved albeit that for some beams the DRP discrepancy is $> \pm 5.0 \%$. Taken in conjunction with reference plan gamma analysis, a $5.0 \%$ absolute difference between baseline DRP discrepancies and adaptive plan DRP discrepancies for a given beam is considered acceptable. On this basis MU2net provides assurance of adaptive online planning and if the plan passes QA, the accuracy of the TPS calculation for these segments on our QA phantom is verified. When MU2net and Monaco have a DRP discrepancy between $5.0 \%-10.0 \%$, this is attributed to the limitation of the MU2net algorithm. Since no evident effect on segment size with DRP discrepancies can be observed. Therefore, reference plan allows us to identify the beams MU2net has more difficulties with to accurately compute the dose for, meaning a smaller than $\pm 5.0 \%$ discrepancy between Monaco and MU2net for that beam will also not expected during adaptive planning. It has been observed that discrepancies between MU2net and Monaco throughout treatments remain within $\pm 5.0 \%$ of those for the reference plan. In addition, after each fraction to the newly created adapted plan undergoes the same QA as the reference plan to independently verify the TPS accuracy.

\section{Conclusion}

The use of MU2net as a secondary dose check for the Elekta Unity MR-Linac has been investigated. For G0 acceptable discrepancies are observed for central axis square fields $<15 \times 15 \mathrm{~cm}^{2}$. However, off-axis fields exhibit greater discrepancies and this requires further investigation outside the scope of this work. Clinically relevant patient specific QA results exhibit differences with the Monaco TPS of $< \pm 10.0 \%$, with most differences for the beams of all plans being less than $< \pm 5.0 \%$. In the clinical workflow MU2net can be used to interrogate adaptive plans and provide a sanity check for Monaco v5.4 dose calculations. However, it is recommended to correlate MU2net DRPs with pre and post treatment dosimetry verification. Given the current limitations of the MU2net algorithm it should be used as an independent dose check utilising baseline data to assess the integrity of adapted plans online. The MU2net dose computation time of approximately $1.0 \mathrm{~min}$ for all clinical treatments reported in this work is acceptable. The inclusion of modelling for the posterior coil and cryostat in the MU2net dose computations would be beneficial.

\section{Declarations}

\section{Funding}

No funding was received to assist with the preparation of this manuscript.

\section{Conflicts of interests/Competing interests}

The authors have no relevant financial or non-financial interests to disclose.

\section{Ethics approval}

This article does not contain any studies with human participants or animals performed by any of the authors. Therefore, no ethics approval was required (HREC Reference number: LNR/2021/QTHS/74993) 


\section{Data Availability}

All data relevant to this article can be made available upon request.

\section{Acknowledgements}

We gratefully acknowledge the assistance of Jason Arts (Elekta), François Husson and Barbara Varenne (DOSIsoft) for their contribution in the preparation of this manuscript.

\section{References}

1. J. J. W. Lagendijk, B. W. Raaymakers, and M. van Vulpen, "The Magnetic Resonance Imaging-Linac System," Semin. Radiat. Oncol., vol. 24, no. 3, pp. 207-209, 2014, doi:

10.1016/j.semradonc.2014.02.009.

2. A. S. Bertelsen et al., "First clinical experiences with a high field 1.5 T MR linac," Acta Oncol. (Madr)., vol. 58, no. 10, pp. 1352-1357, 2019, doi: 10.1080/0284186X.2019.1627417.

3. J. Wang, J. Yung, M. Kadbi, K. Hwang, Y. Ding, and G. S. Ibbott, "Assessment of image quality and scatter and leakage radiation of an integrated MR-LINAC system:", Med. Phys., vol. 45, no. 3, pp. 1204-1209, 2018, doi: 10.1002/mp.12767.

4. L. I. Cerviño, J. Du, and S. B. Jiang, "MRI-guided tumor tracking in lung cancer radiotherapy.," Phys. Med. Biol., vol. 56, no. 13, pp. 3773-3785, Jul. 2011, doi: 10.1088/0031-9155/56/13/003.

5. S. B. Ahmad et al., "Evaluation of a commercial MRI Linac based Monte Carlo dose calculation algorithm with geant 4," Med. Phys., vol. 43, no. 2, pp. 894-907, 2016, doi: 10.1118/1.4939808.

6. M. Friedel, M. Nachbar, D. Mönnich, O. Dohm, and D. Thorwarth, "Development and validation of a 1.5 T MR-Linac full accelerator head and cryostat model for Monte Carlo dose simulations," Med. Phys., vol. 46, no. 11, pp. 5304-5313, 2019, doi: 10.1002/mp.13829.

7. S. J. Woodings et al., "Beam characterisation of the 1.5 T MRI-linac," Phys. Med. Biol., vol. 63, no. 8, 2018, doi: 10.1088/1361-6560/aab566.

8. D. Winkel et al., "Adaptive radiotherapy: The Elekta Unity MR-linac concept," Clin. Transl. Radiat. Oncol., vol. 18, no. xxxx, pp. 54-59, 2019, doi: 10.1016/j.ctro.2019.04.001.

9. J. B. Smilowitz et al., "AAPM Medical Physics Practice Guideline 5.a.: Commissioning and QA of Treatment Planning Dose Calculations - Megavoltage Photon and Electron Beams," J. Appl. Clin. Med. Phys., vol. 17, no. 1, p. 6166, 2016, doi: 10.1120/jacmp.v17i1.6166.

10. M. R. Malisan, "MU Calculation : the ESTRO formalism," no. April, 2017.

11. S. A. Graves et al., "Commissioning and performance evaluation of RadCalc for the Elekta unity MRIlinac," J. Appl. Clin. Med. Phys., vol. 20, no. 12, pp. 54-62, 2019, doi: 10.1002/acm2.12760.

12. Dosimetry of Small Static Fields Used in External Beam Radiotherapy, no. 483. Vienna: INTERNATIONAL ATOMIC ENERGY AGENCY, 2017. 
13. A. Micke, D. F. Lewis, and X. Yu, "Multichannel film dosimetry with nonuniformity correction," Med. Phys., vol. 38, no. 5, pp. 2523-2534, 2011, doi: 10.1118/1.3576105.

\section{Figures}

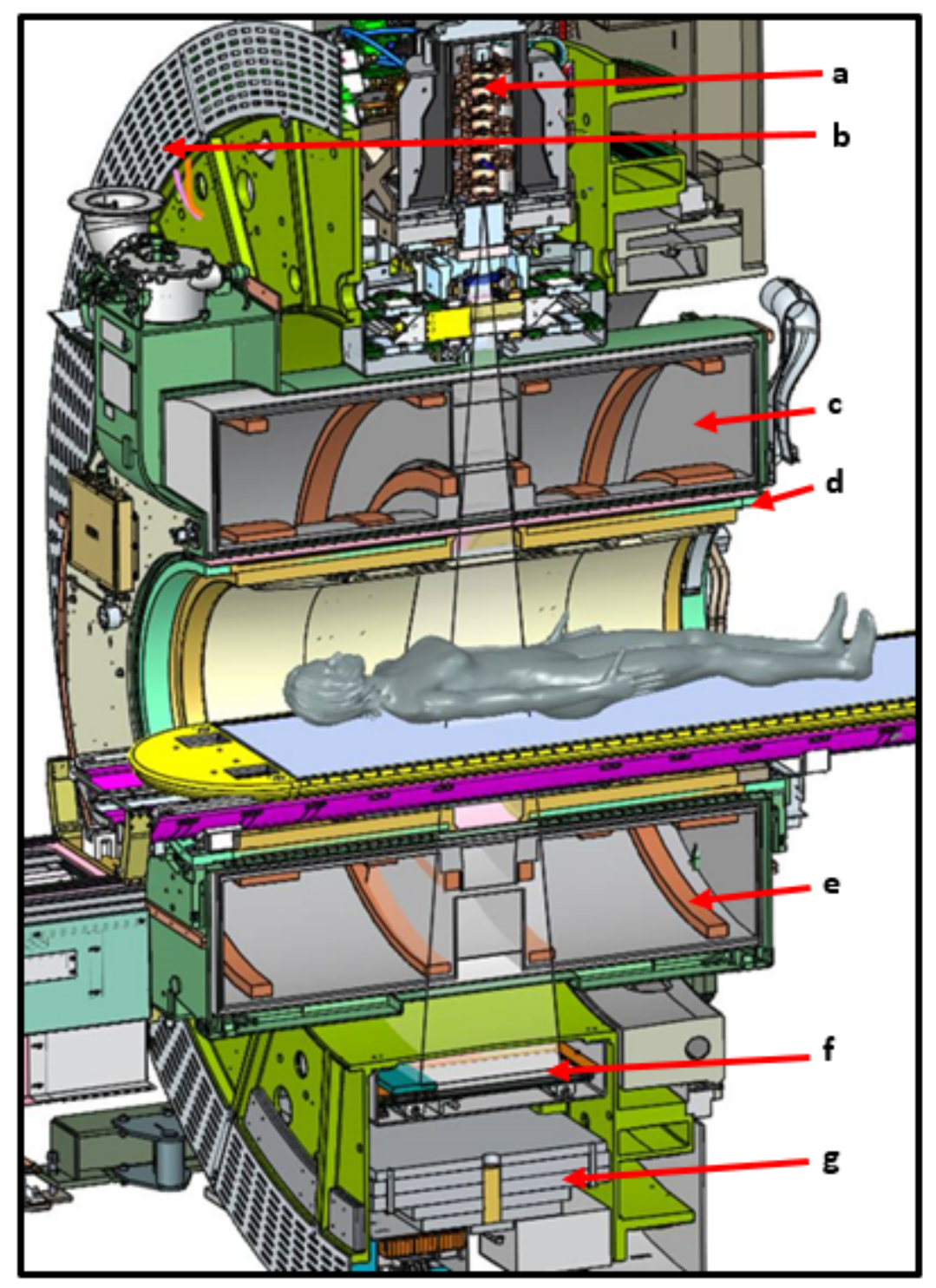

\section{Figure 1}

MR Linac; a) accelerating waveguide, b) rotating gantry, c) cryostat, d) gradient coils, superconducting coils and system body coil (QBC), e) active shielding coils, f) EPID, g) beam stopper (courtesy of Elekta) 


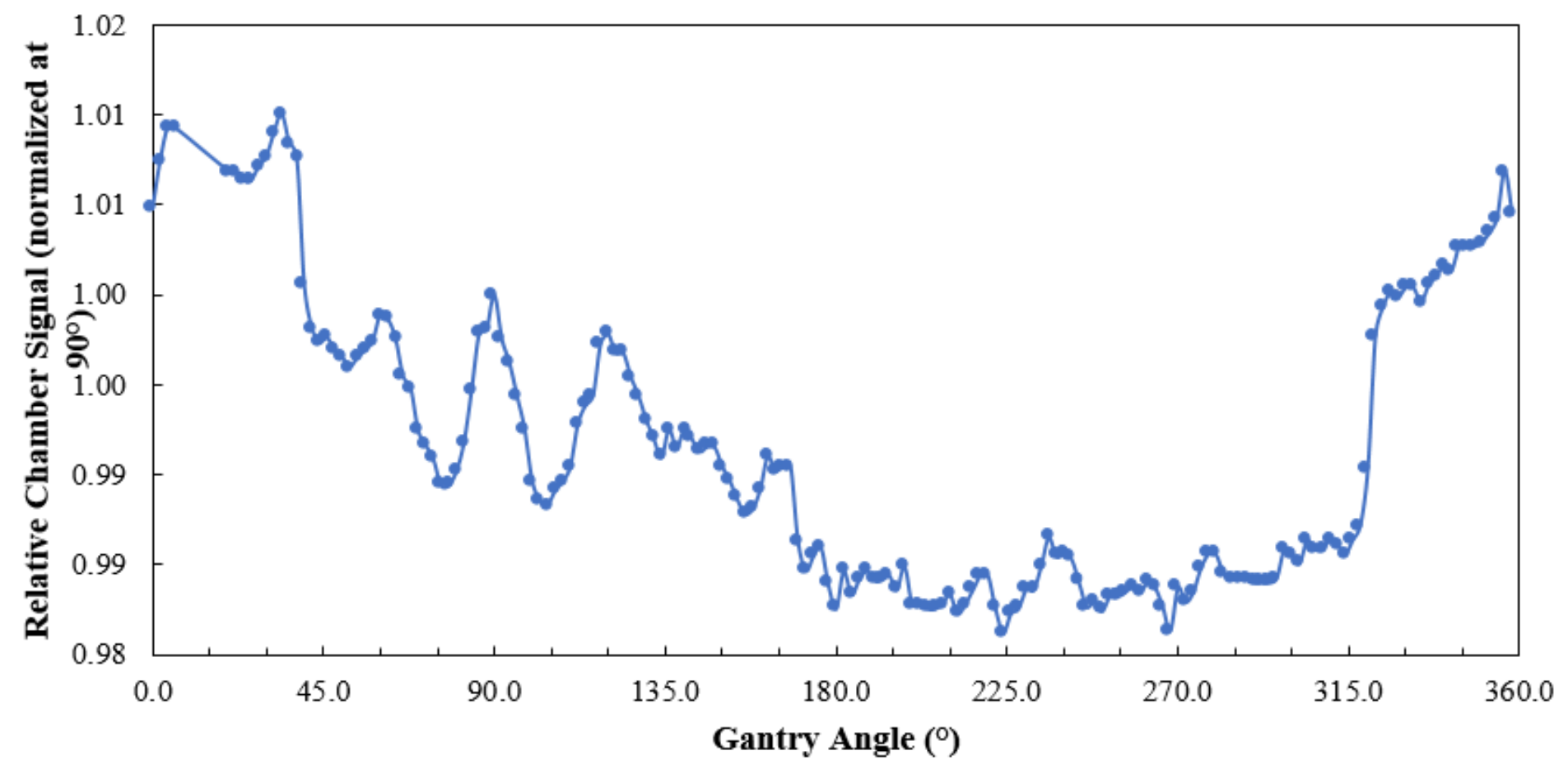

Figure 2

Relative cryostat transmission characterisation curve, normalized to G90 (courtesy of Elekta)

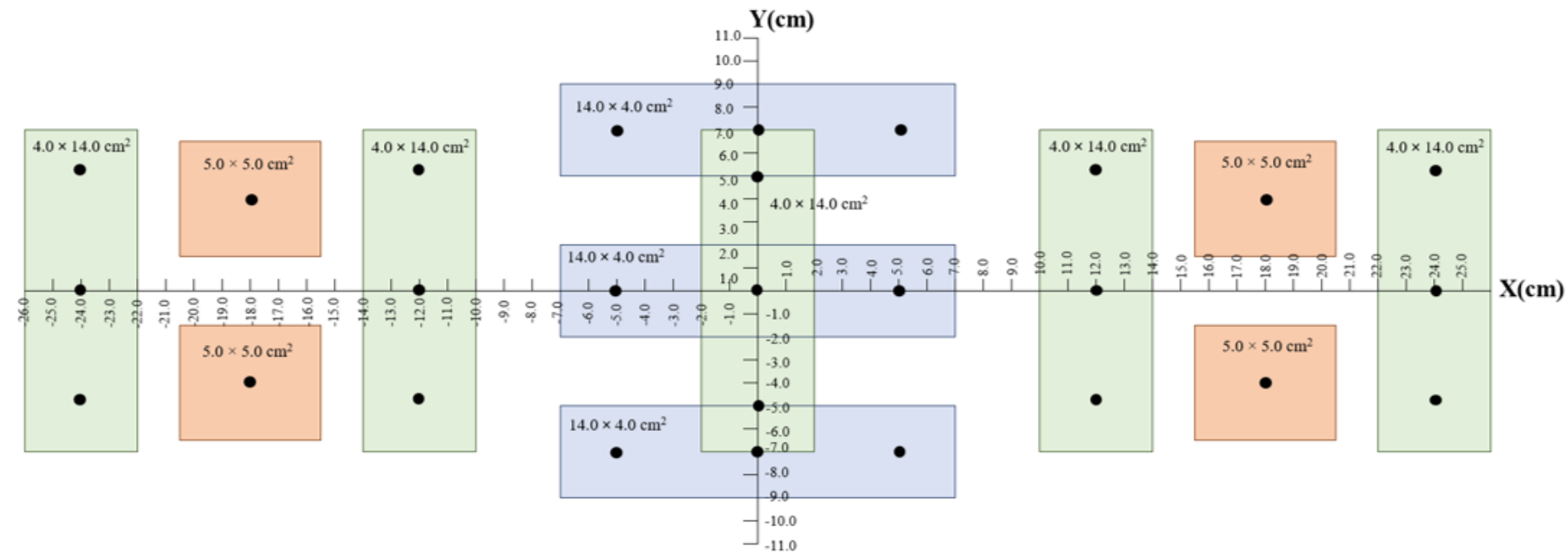

Figure 3

BEV of custom fields, where the dot $(\bullet)$ represents the chamber measurement position 


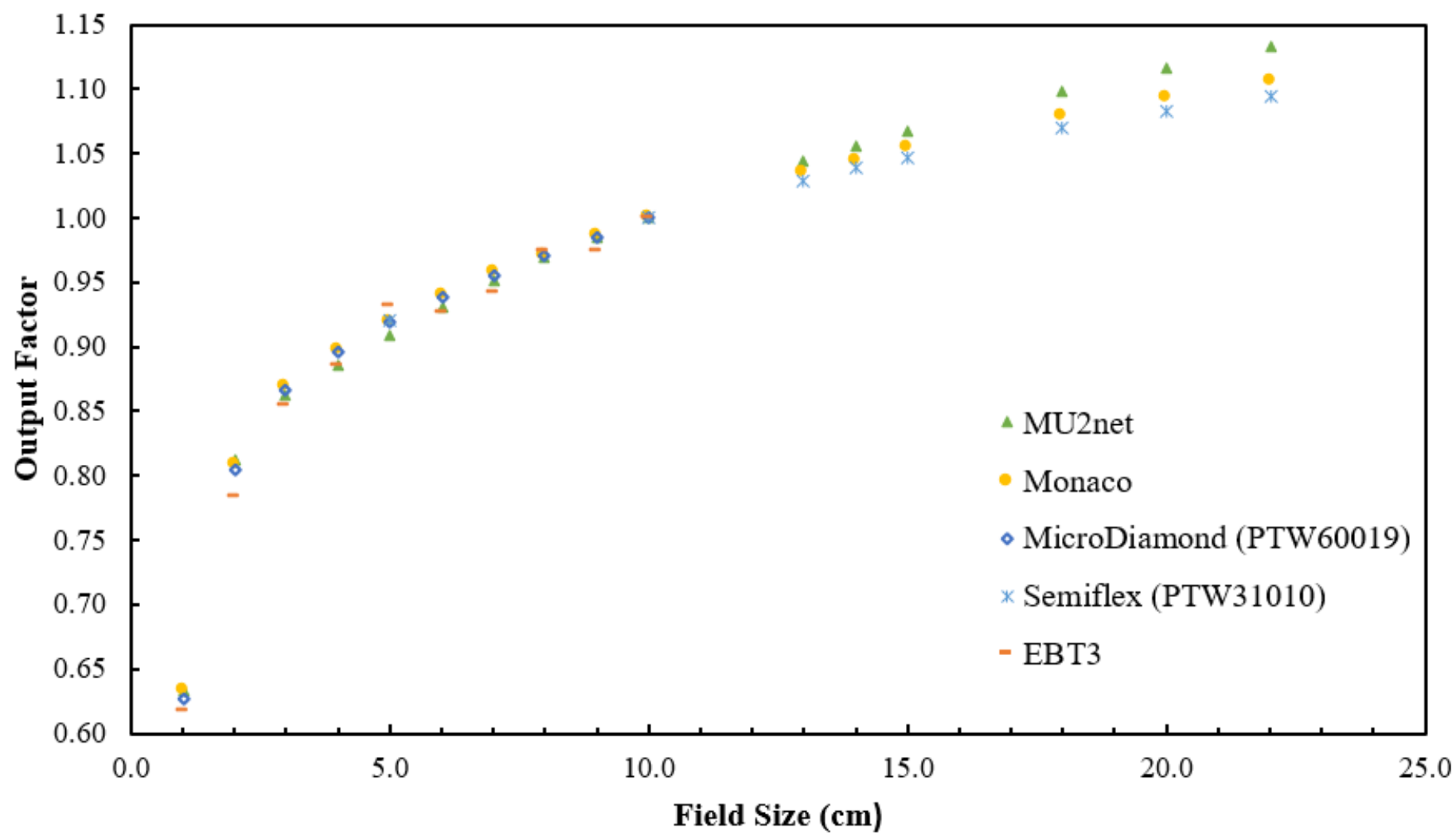

Figure 4

Output factors for MU2net, Monaco, measured (MicroDiamond and Semiflex) and EBT3 at SSD = 133.5 $\mathrm{cm}$ 


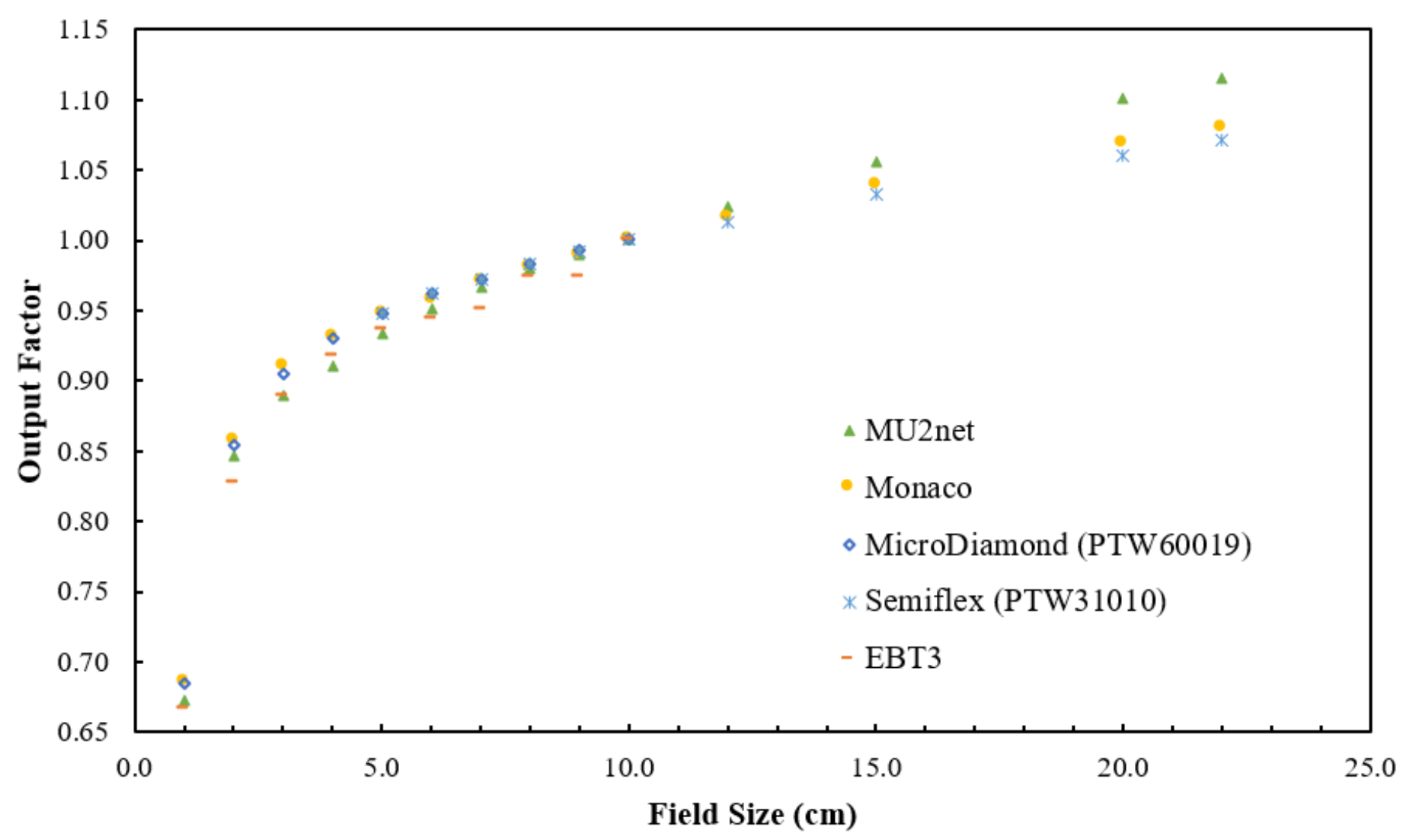

Figure 5

Output factors for MU2net, Monaco, measured (MicroDiamond and Semiflex) and EBT3 at SSD = 138.5 $\mathrm{cm}$ 


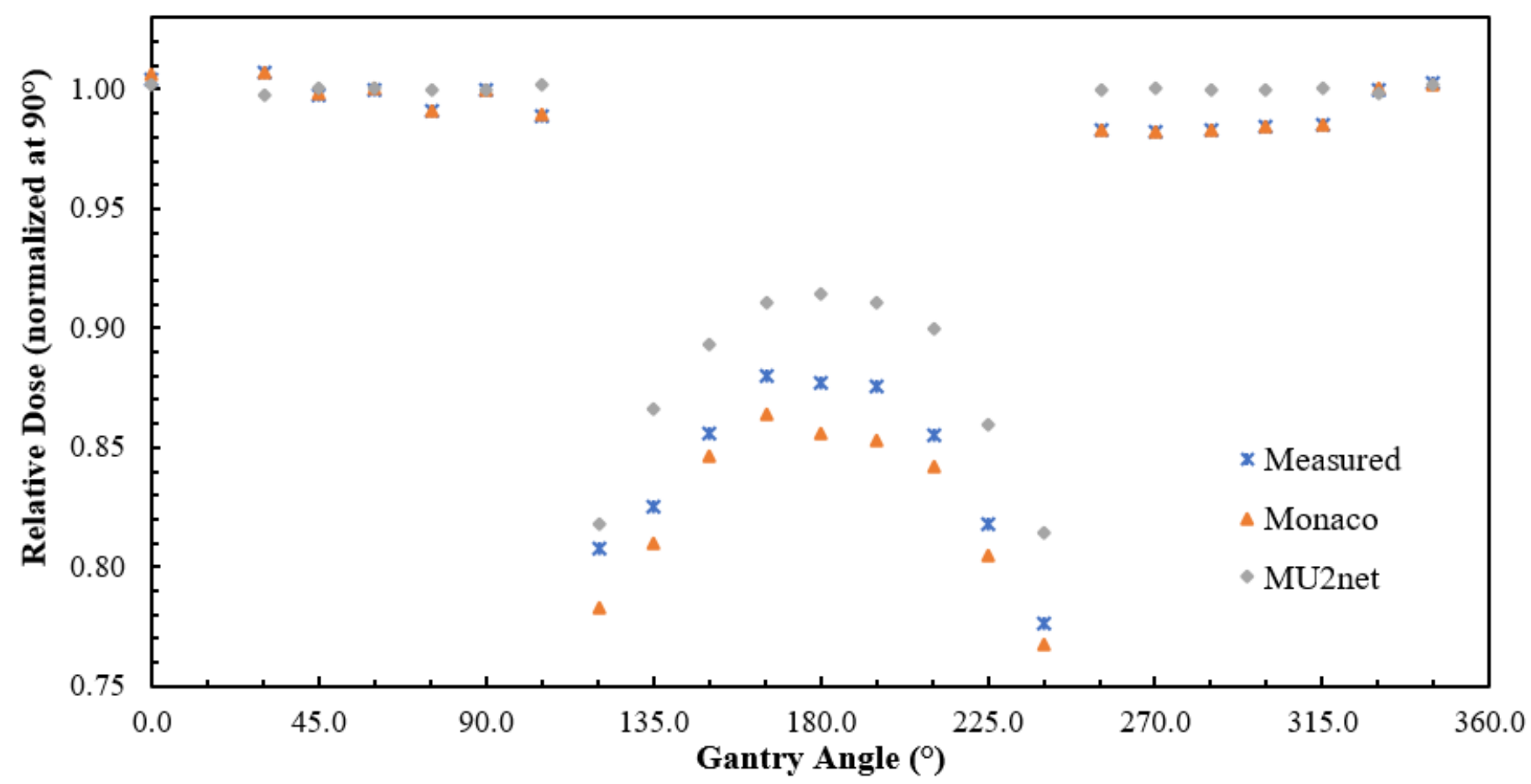

Figure 6

Directional dependence of measured output, normalized to G90, compared to Monaco and MU2net

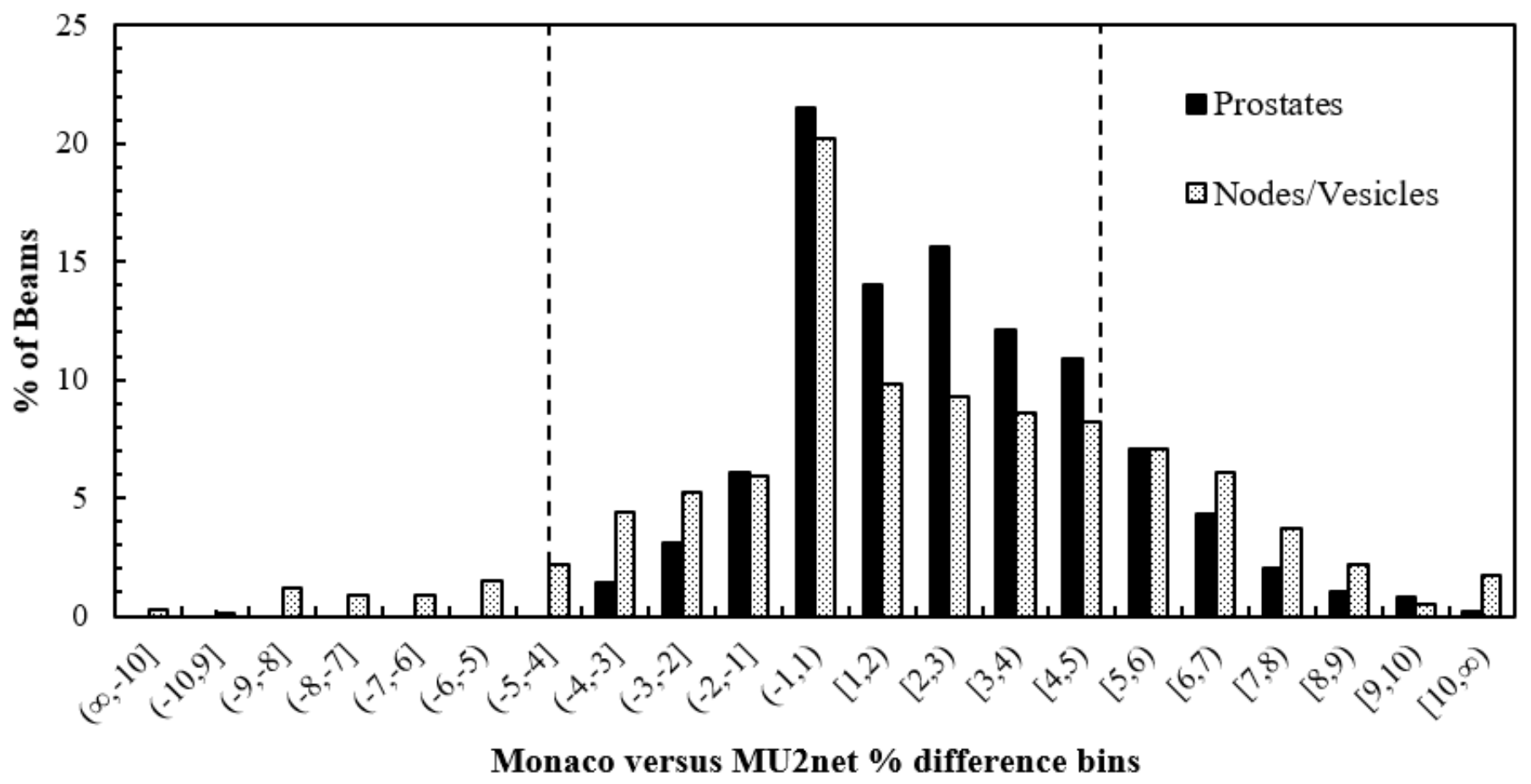

Figure 7 
Monaco versus MU2net percentage differences for individual beams. The two categories are the prostate fields (black) and the nodes/vesicles (dotted)

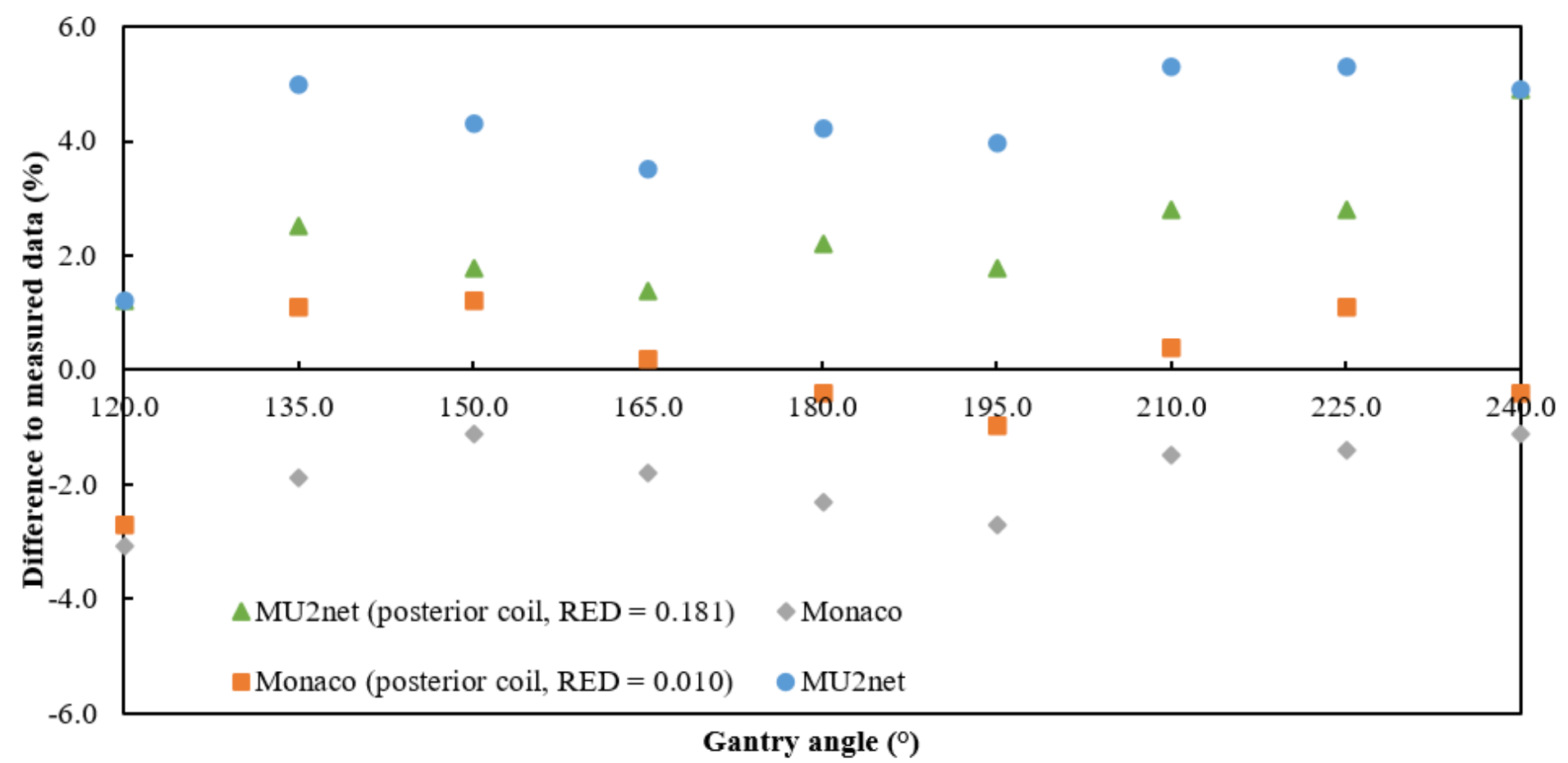

Figure 8

Percentage difference of angular dependence of measured output compared to Monaco with and without a RED 0.010 for the posterior coil and MU2net with and without the posterior coil 